barbed versus standard suture for laparoscopic vaginal cuff closure and found small and nonsignificant differences in operative time, cuff healing, dyspareunia, and sexual function ( $J$ Minim Invasive Gynecol. 2013;20(4):492-498).

Now that the authors of the abstracted study have established the possibility of a clinically important difference in sacral colpopexy suture integrity, a randomized trial can be designed varying suture materials experimentally and following patients to clinical failure. Imaging to assess mesh separation could also be included. Although randomization to air knots would not pass institutional review, there would likely be real-world variation in knot-tissue approximation in each suture material group, particularly if trainees are participating.-LAL)

\title{
Assessment of Voiding After Sling: A Randomized Trial of 2 Methods of Postoperative Catheter Management After Midurethral Sling Surgery for Stress Urinary Incontinence in Women
}

\author{
Elena Tunitsky-Bitton, Alana Murphy, Matthew D. Barber, Howard B. Goldman, \\ Sandip Vasavada, and J. Eric Jelovsek \\ Women's Health Institute, and Obstetrics and Gynecology (E.T.-B., M.D.B., J.E.J.) and Glickman Urological Institute (A.M., \\ H.B.G., S.V.), Division of Female Pelvic Medicine and Reconstructive Pelvic Surgery, Cleveland Clinic, Cleveland, OH
}

Am J Obstet Gynecol 2015;212:597.el-597.e9

\begin{abstract}
Indwelling catheters are the leading cause of hospital-acquired urinary tract infections and are a source of discomfort, embarrassment, and inconvenience for patients. An assessment of voiding function (voiding trial) after anti-incontinence surgery is often performed to determine which patients can be safely discharged without a catheter. The backfill standard voiding trial (SVT) is a commonly used method to evaluate postoperative urinary retention. Standard voiding trial provides an objective assessment of postoperative voiding function. It involves filling the bladder with $300 \mathrm{~mL}$ of sterile fluid via the catheter in the recovery room, removing the catheter, allowing the patient to void, and measuring the voided urine. If the voided amount is more than two-thirds the infused volume, the patient is discharged without an indwelling catheter. In an alternative subjective method, a visual analog scale is used to determine the urinary force of stream (FOS) before and after surgery. The criteria for discharge without a catheter with this method are a report by the patient that her FOS is $50 \%$ or greater of her baseline FOS regardless of the amount voided. The FOS voiding trial appears to be safe, but it is not known whether a patient's subjective perception of voiding correlates with SVT results.

The aim of this double-blind randomized comparative trial was to determine if the subjective patient evaluation with the FOS voiding trial is a safe method to determine the need for indwelling catheterization after outpatient sling surgery. The authors tested the hypothesis that the rate of catheterization would be significantly lower when using the FOS technique as compared with the backfill SVT. Patients in both groups had undergone an outpatient midurethral sling procedure for female stress urinary incontinence without any other concomitant surgery. All participants underwent the same standardized postoperative protocol in the recovery room.

Participants were interviewed and completed questionnaires at 4 time points: preoperatively and 2 to 4 days, 7 to 9 days, and 6 weeks postoperatively. The primary study outcome was the rate of catheterization within 6 weeks after the surgery. All postoperative interviews assessed pain, tolerance of physical activity, urinary FOS, and satisfaction with the surgery. Urinary symptoms were evaluated by use of validated questionnaires (Incontinence Severity Index and Urinary Distress Inventory, Short Form) before the surgery and 6 weeks postoperatively.
\end{abstract}


Six-week follow-up data were available for 102 of the 108 participants who had enrolled and were randomized (50 in the FOS trial and 52 in the SVT). Demographic characteristics and urinary symptoms were similar in the 2 groups. The catheterization rate was similar in the 2 groups (FOS 13 [26\%], SVT 13 [25.5\%]; $P=0.95$ ). There was a moderate correlation between the 2 groups for the amount voided (Spearman $\rho=0.5 ; P<0.001$ ). Postoperative evaluation showed no significant group differences in mean catheter days, pain scores, Incontinence Severity Index, and Urinary Distress Inventory, Short Form scores. None of the patients discharged home without a catheter in either group required catheter reinsertion within 6 weeks after the surgery.

Subjective evaluation of the FOS is a reliable and safe method to assess continence after outpatient midurethral surgery. There was no difference in catheterization rate between these 2 voiding trials, and the patient's subjective assessment of FOS correlated well with the measured voided amount in the SVT.

\section{EDITORIAL COMMENT}

(One of the best ways to improve both efficiency and quality of perioperative care is to find ways to allow providers to safely accomplish the same or better level of care in less time or with less effort. This randomized trial provides compelling data of one such way. As the authors point out, midurethral slings are 1 of the most common surgeries performed in the United States. Slings provide similar efficacy of treatment of stress urinary incontinence to other, more historical surgical therapies, with shorter hospital stay, easier recovery, and less blood loss. Nonetheless, 1 of the most common complications after sling surgery is voiding dysfunction, with a large minority of women requiring catheter drainage of the bladder for some period postoperatively. Quickly and accurately determining who needs a catheter after discharge is an important component of efficient care.

Other randomized trials have shown that the backfill method, where saline is instilled retrograde into the postoperative catheter, which is then removed so the patient can test voiding, is faster than spontaneous bladder filling, does not require additional catheterization, is well accepted by patients, and does not alter the rate of needing a catheter (Am J Obstet Gynecol. 2007;197: 627.e1-627.e4). Generally after a backfill, the patient's bladder will be scanned with bedside ultrasound to determine the residual volume to determine whether a temporary catheter is needed. This trial takes these improvements one step further by testing the use of the patient's own assessment to determine whether the catheter is needed. This FOS method of determining whether a patient needs a catheter prior to discharge would then eliminate the need for bladder scan for residual volume or in- and out-catheterization to measure the residual directly. This eliminates 1 more step or procedure standing between the patient and a safe discharge. In this trial, all subjects underwent procedures designed to simulate both methods of void trial, backfill, and FOS. The randomization was with regard to which method's findings were used to determine whether the patient "passed" or "failed" and whether she was discharged with a catheter. Double blinding was accomplished by masking the patient and the entire care team, with the exception of 1 perioperative nurse opening the envelope, to the treatment assignment.

The authors clearly document that their groups had similar rates of catheterization as well as similar low rates of needing a catheter in the postoperative period after having been judged as "passed." Satisfaction, pain, urinary urgency, and other assessments were all similar between groups, supporting the veracity of the FOS method. Most of these effects are a function of the improvement in quality of life after the incontinence surgery, rather than the voiding method utilized, but it is reassuring that the findings are the same in both groups. The FOS assigned by the patient correlated moderately with the voided volume. It seems strange that the article does not report length of stay postoperatively, because part of the appeal of the FOS method is to decrease the intervention involved in determining which patient needs a catheter and thereby increase efficiency of postoperative care, but the main goal of the trial was to confirm the utility and safety of the method versus current practice. These findings also may not be generalizable to other women having additional pelvic floor reconstructive surgeries at the time of sling, or other gynecologic procedures in general, because this trial studied only women having midurethral slings as an isolated procedure.-ACW) 